Cite this: Phys. Chem. Chem. Phys., 2012, 14, 4196-4203

\title{
Salt-regulated attraction and repulsion of spherical polyelectrolyte brushes towards polyelectrolyte multilayers $\dagger$
}

\author{
Christoph Hanske, ${ }^{a}$ Christian Schneider, ${ }^{b c}$ Markus Drechsler, ${ }^{d}$ \\ Alexander Wittemann ${ }^{b e}$ and Andreas Fery ${ }^{* a}$
}

Received 28th October 2011, Accepted 4th February 2012

DOI: $10.1039 / \mathrm{c} 2 \mathrm{cp23408d}$

Adsorption of colloidal particles presents an interesting alternative to the modification of surfaces using covalent coupling or physisorption of molecules. However, to tailor the properties of these materials full control over the effective particle-substrate interactions is required. We present a systematic investigation of the adsorption of spherical polyelectrolyte brushes (SPB) onto polyelectrolyte multilayers (PEM). A brush layer grafted from colloidal particles allows the incorporation of various functional moieties as well as the precise adjustment of their adsorption behaviour. In the presence of oppositely charged surfaces the amount of adsorbed SPB monotonically increases with the ionic strength, whereas equally charged substrates efficiently prevent colloidal attachment below a threshold salt concentration. We found that the transition from the osmotic to the salted brush regime at approximately $100 \mathrm{mM}$ coincided with a complete loss of substrate selectivity. In this regime of high ionic strength, attractive secondary interactions become dominant over electrosteric repulsion. Due to the soft polyelectrolyte corona a surface coverage exceeding the theoretical jamming limit could be realized. Both the adsorption kinetics and the resulting thin film morphologies are discussed. Our study opens avenues for the production of two-dimensional arrays and three-dimensional multilayered structures of SPB particles.

\section{Introduction}

A modular approach to surface modification based on the physisorption of components from solution is advantageous because it avoids any covalent chemical coupling. One of the best studied examples using molecular components is the layer-by-layer (LbL) deposition of oppositely charged polyelectrolytes. ${ }^{1-4}$ In combination with spray or dip coating, it can be applied to large substrates and the non-covalent nature of the underlying interactions allows for a broad spectrum of potential components. ${ }^{5-7}$ Already in the pioneering work of Iler on LbL assembly, colloidal particles were considered as potential building blocks for such films. ${ }^{8}$ Various examples show that functional colloidal particles immobilized on solid substrates are promising building blocks for optical, electronic

${ }^{a}$ Physical Chemistry II, University of Bayreuth, Universitätstrasse 30, 95440 Bayreuth, Germany.E-mail: andreas.fery@uni-bayreuth.de

${ }^{b}$ Physical Chemistry I, University of Bayreuth, Universitätstrasse 30, 95440 Bayreuth, Germany

${ }^{c}$ F-I2 Soft Matter and Functional Materials, Helmholtz-Zentrum

Berlin, Hahn-Meitner-Platz 1, 14109 Berlin, Germany

${ }^{d}$ Macromolecular Chemistry II, University of Bayreuth, Universitätstrasse 30, 95440 Bayreuth, Germany

${ }^{e}$ Department of Chemistry, University of Constance,

Universitätstrasse 10, 78464 Konstanz, Germany

$\dagger$ Electronic supplementary information (ESI) available. See DOI: $10.1039 / \mathrm{c} 2 \mathrm{cp} 23408 \mathrm{~d}$ or magnetic devices., ${ }^{9,10}$ The efficiency of these materials depends on the physical properties of the colloids as well as on their spatial arrangement on the substrate. Therefore, there is a strong demand for functional particles whose interaction with a given substrate can be precisely tuned by external stimuli.

Brush covered colloids are very attractive building blocks for such coatings because the brush layer moderates the interaction with the substrate independent of the type of particles. ${ }^{11}$ Herein, we focus on the interaction of spherical polystyrene particles bearing dense polystyrene sulfonate (PSS) brushes and polyelectrolyte multilayer coated substrates. Because the interactions are solely determined by the outermost layer, our approach can be employed to any type of colloids including inorganic and polymer particles. Moreover, the polyelectrolyte brush layer adds electrosteric stabilization and functionalities such as stimuli-responsiveness, compatibility, adhesiveness or non-adhesiveness to carrier particles that could exhibit special optical, mechanical or magnetic features. In solution, the properties of the SPB are widely determined by the confinement of the counterions of the polyelectrolyte chains. At low concentrations of added salt, approximately $95 \%$ of the counterions of the polyelectrolyte chains are trapped within the brush creating a huge osmotic pressure. ${ }^{12,13}$ This results in a marked stretching of the polyelectrolyte chains. The responsiveness towards external stimuli such as the ionic strength and $\mathrm{pH}$ allows precise control over the 
expansion of the brush layer in solution. ${ }^{14}$ Beyond stabilization of particles through a surrounding polyelectrolyte brush, the brush layer may serve as a carrier for active nanostructures, namely for metal nanoparticles, enzymes, and conductive polymers yielding functional colloids with tailored biological, catalytic or electronic properties. ${ }^{15-17}$ Further functionalization is accomplished by loading the core with hydrophobic substances, including many drugs. The composite particles are stable against coagulation and can be easily handled. ${ }^{18}$

Despite the technological potential of SPB in functional surface coatings, not much effort has been devoted yet to study their interactions with solid substrates. Preceding studies using negatively charged mica surfaces demonstrated distinct differences in the adsorption behaviour of cationic and anionic SPB. ${ }^{19,20}$ Whereas anionic SPB exhibited a high lateral mobility during drying resulting in hexagonally packed arrangements, cationic SPB were strongly affixed to the surface, which resulted in open structures. Intriguingly, the force of adhesion could be adjusted by the ionic strength of the surrounding medium. ${ }^{19}$ We extend these studies to substrates coated with charged polymers. Polyelectrolyte multilayers are applicable to almost any solid support and the layer-by-layer technique is compatible with a wide selection of polymers. ${ }^{21}$ The assembly process even allows the incorporation of charged particles opening avenues to highly functional surfaces. ${ }^{22,23}$ Recent works based on $\mathrm{pH}$ and thermoresponsive block copolymer micelles demonstrated that stimuli-responsiveness can be introduced into these films as well. ${ }^{24,25}$

To tailor the properties of such functional surfaces, a profound understanding of the particle adsorption behaviour is obligatory. Here we present a detailed investigation of SPB adsorption on polyelectrolyte multilayers of opposite and equal charge. We used alternating layers of polystyrene sulfonate (PSS) and poly(diallyldimethylammonium chloride) (PDA). These polyelectrolytes form stable multilayers over a wide range of ionic strengths (see ESI $\dagger$ ). Depending on the terminating layer the surface potential can be switched between negative (PSS) and positive (PDA) without significantly altering the wettability. ${ }^{26}$ To ensure compatibility with the substrate, polystyrene particles coated with spherical PSS brushes were used. Exclusive use of strong polyelectrolytes is to render the spatial dimensions of the brush layer and their interactions with the polyelectrolyte multilayer independent of the $\mathrm{pH}$ value of the bulk solution, which makes sure that the interactions are governed by the concentration of added salt.

The kinetics of SPB adsorption is monitored over a wide range of salt concentrations, both under electrostatically attractive and repulsive conditions. We then compare the saturation coverage of the substrate with theoretical predictions based on the Random Sequential Adsorption model for hard spheres.

\section{Experimental section}

\section{Particles}

The synthesis of the SPB particles and their characterization in solution are described in detail in ref. 27. Briefly, PSS brushes were grafted from polystyrene particles bearing covalently anchored photoinitiator moieties by UV induced polymerization of sodium styrene sulfonate. The narrowly dispersed polystyrene cores have an average radius of $126 \pm 2 \mathrm{~nm}$ as measured by dynamic light scattering (DLS). The hydrodynamic thickness of the PSS brush is $74 \pm 3 \mathrm{~nm}$ in water. The PSS chains were cleaved off the surface of the cores and analyzed by size exclusion chromatography. The molecular weight of the longest chains that govern the spatial extension of the polyelectrolyte brush was determined as $67600 \pm 4950 \mathrm{~g} \mathrm{~mol}^{-1}$ corresponding to a contour length of $82 \pm 6 \mathrm{~nm} \cdot{ }^{27}$ The polydispersity of the chains given as weight-averaged molecular weight by the number-averaged molecular weight is 2.1 , which is a common value for polymer chains prepared by free radical polymerization. The grafting density on the particle surface is $0.03 \pm 0.01$ chains per $\mathrm{nm}^{2} \cdot{ }^{27}$ Purification of the SPB latex was accomplished by exhaustive ultrafiltration against deionized water taken from a reverse osmosis water purification system (Millipore Academic A10).

\section{Polyelectrolyte solutions}

All polyelectrolytes were purchased from Sigma Aldrich and diluted to $1 \mathrm{~g}^{-1}$ in water. The polyelectrolyte solutions for spray coating contained $50 \mathrm{mM}$ of $\mathrm{NaCl}(99.88 \%$, Fisher Scientific). PEI: poly(ethylene imine), $M_{\mathrm{W}}=25000 \mathrm{~g} \mathrm{~mol}^{-1}$; PSS: poly(sodium-4-styrene sulfonate), $M_{\mathrm{W}}=70000 \mathrm{~g} \mathrm{~mol}^{-1}$; PDA: poly(diallyldimethylammonium chloride), $M_{\mathrm{W}}=$ 100 000-200000 $\mathrm{g} \mathrm{mol}^{-1}$.

\section{Substrate preparation}

Silicon wafers were cut into pieces of $1 \mathrm{~mm} \times 1.5 \mathrm{~mm}$ and cleaned by the RCA method using analytical grade chemicals (2-propanol, $\mathrm{NH}_{3}, \mathrm{H}_{2} \mathrm{O}_{2}$ from VWR). ${ }^{28}$ An adhesion promoting layer was deposited by immersing the substrates into a solution of PEI for $30 \mathrm{~min}$. The build-up of (PSS/PDA) and (PSS/PDA) 5.5 multilayers by spray coating followed the procedure reported in ref. 5 .

\section{Particle deposition}

For the adsorption experiments SPB suspensions with a particle content of $0.1 \mathrm{wt} \%$ and $\mathrm{NaCl}$ concentrations between $0.1 \mathrm{mM}$ and $1000 \mathrm{mM}$ were prepared. Loose aggregates could be dispersed by $2 \mathrm{~min}$ of sonication in an ultrasonic cleaner (Elmasonic S30H). Subsequently, PEM coated silicon wafers were immersed into these suspensions. After defined exposure times the substrates were withdrawn and dipped twice into saline solutions with ionic strengths corresponding to those of the suspensions. This quenching procedure was followed by threefold dipping in deionized water and drying in a $\mathrm{N}_{2}$ stream. In situ imaging of wet surfaces clearly demonstrated that the drying process does not affect the total amount of adsorbed particles as long as excess particles were removed from the suspension before drying. However, it has to be noted that weakly adsorbed particles can rearrange within the adsorbed layer during drying.

\section{AFM measurements and image analysis}

Imaging in air was performed with a Dimension IIIa atomic force microscope (Veeco Instruments) operated in tapping mode. 
Silicon cantilevers (OMCL-AC160TS-W2, Olympus) with a typical force constant of $42 \mathrm{~N} \mathrm{~m}^{-1}$ and a resonance frequency around $300 \mathrm{kHz}$ were used. In situ images were recorded in deionized water. For this purpose a NanoWizard AFM (JPK Instruments) and soft cantilevers (CSC17, MikroMasch) with a spring constant of $0.15 \mathrm{~N} \mathrm{~m}^{-1}$ and a resonance frequency of $12 \mathrm{kHz}$ were utilized. Scans of $10 \mu \mathrm{m} \times 10 \mu \mathrm{m}$ sections were recorded at three or more positions per substrate. After manual background correction and grey scale adjustment in Gwyddion the images were subjected to the automated particle counting procedure implemented in ImageJ. As the particle recognition algorithm responds sensitively to the presence of three-dimensional agglomerates, only images with little or no aggregation were used for the evaluation of the surface coverage.

\section{Cryogenic TEM}

Cryo-TEM samples were prepared as described in ref. 29. A thin film of the SPB suspension was spread on a hydrophilized lacey carbon TEM grid (mesh size 200, Plano GmbH, Germany) and vitrified in liquid ethane. The contrast of the brush was enhanced by counterion exchange with $\mathrm{CsCl}$ and adsorption of the protein bovine serum albumin (BSA). Imaging was carried out with a Zeiss EM922 OMEGA EFTEM (Zeiss NTS GmbH, Germany) in combination with a bottom-mounted CCD camera system (UltraScan 1000, Gatan, Germany). An acceleration voltage of $200 \mathrm{kV}$ was used during the measurement conducted at a temperature of $90 \mathrm{~K}$.

\section{Dynamic light scattering}

The hydrodynamic radii of the particles were measured with a setup consisting of an ALV/DLS-5000 goniometer system with a thermostated cis-decaline bath, a He-Ne-laser and an ALV-6010/160 External Multiple Tau Digital Correlator. Suspensions of the SPB were strongly diluted with saline solutions and filtered through syringe filters (Pall Acrodisc, $0.8 \mu \mathrm{m}$ supor membrane) into glass cuvettes. The measurements were performed at a fixed angle of $90^{\circ}$. Hydrodynamic radii were obtained from a cumulant analysis of the correlation functions.

\section{Results and discussion}

\section{Particle morphology in solution}

Since our SPB are composed of the strong polyelectrolyte PSS the degree of deprotonation inside the brush is widely independent of $\mathrm{pH} .{ }^{12}$ As depicted in Fig. 1 the particle morphology in solution is governed by the ionic strength. In the presence of small amounts of monovalent salts the polyelectrolyte chains are strongly stretched due to the high osmotic pressure inside the brush. With increasing salt concentration a pronounced collapse of the shell can be observed by dynamic light scattering (Fig. 1B). The brush thickness in solution is precisely tuneable via the ionic strength and follows the established scaling law. ${ }^{12}$ A similar adjustability is expected regarding the adsorption behaviour.

\section{Adsorption kinetics}

Because of the particle dimensions in the submicron regime, AFM was chosen to investigate the SPB adsorption process. For this purpose we immersed multilayer coated substrates in suspensions of SPB particles. After defined exposure times the samples were washed with deionized water and dried in a nitrogen stream. Fig. 2 shows a typical series of images obtained for the adsorption on PDA at an ionic strength of $100 \mathrm{mM}$. Within the first minutes the number of adsorbed particles increases rapidly. After approximately $60 \mathrm{~min}$ the final saturation coverage is reached. The electrosteric repulsion between the SPB in solution and those adsorbed to the surface causes the formation of a submonolayer of particles. Due to attractive capillary forces that occur during drying of the suspension, island formation is observed at high surface coverage.

The fast quenching procedure combined with the high resolution of AFM allows for a quantitative analysis of the adsorption kinetics. We varied the deposition time from $30 \mathrm{~s}$ up to $60 \mathrm{~min}$ and evaluated the number of adsorbed particles from AFM images of the dried samples. Both PDA and PSS terminated multilayers were used. Fig. 3 displays the evolution of the average particle density adsorbed on PDA as a function of time for ionic strengths ranging from $0.1 \mathrm{mM}$ to $100 \mathrm{mM}$. In the presence of oppositely charged surfaces, SPB adsorption
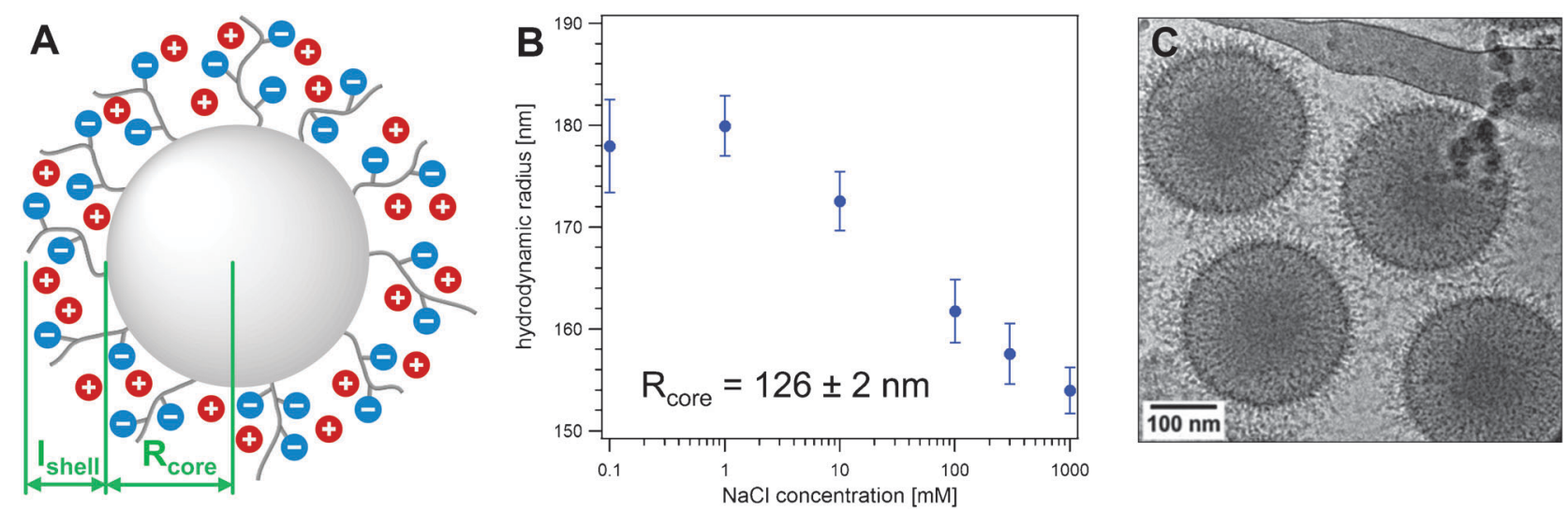

Fig. 1 Schematic depiction of the particle core-shell morphology (A). Counterion condensation inside the brush layer partially compensates the charge of the densely grafted polyanion chains. The thickness of this layer depends strongly on the concentration of added salt (B). The polyelectrolyte brush is clearly visible in Cryo-TEM micrographs of the SPB (C). 

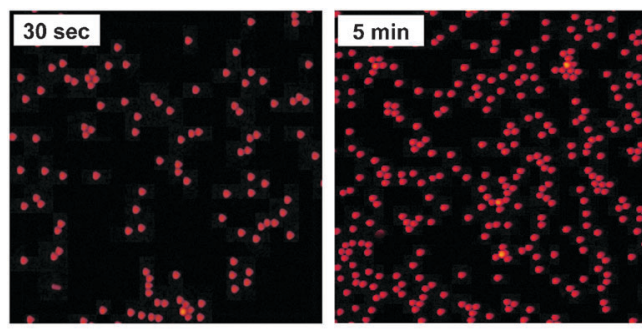

$0.50 \mu \mathrm{m}$

0.45
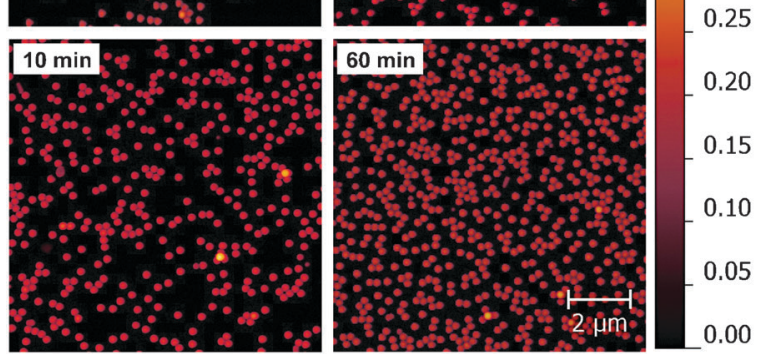

Fig. 2 AFM images of anionic SPB particles adsorbed onto polyelectrolyte multilayer coated substrates for varying adsorption times. The scans show areas of $10 \mu \mathrm{m} \times 10 \mu \mathrm{m}$ and were recorded with dried samples. Particle deposition was performed at an ionic strength of $100 \mathrm{mM}$ on PDA terminated multilayers.

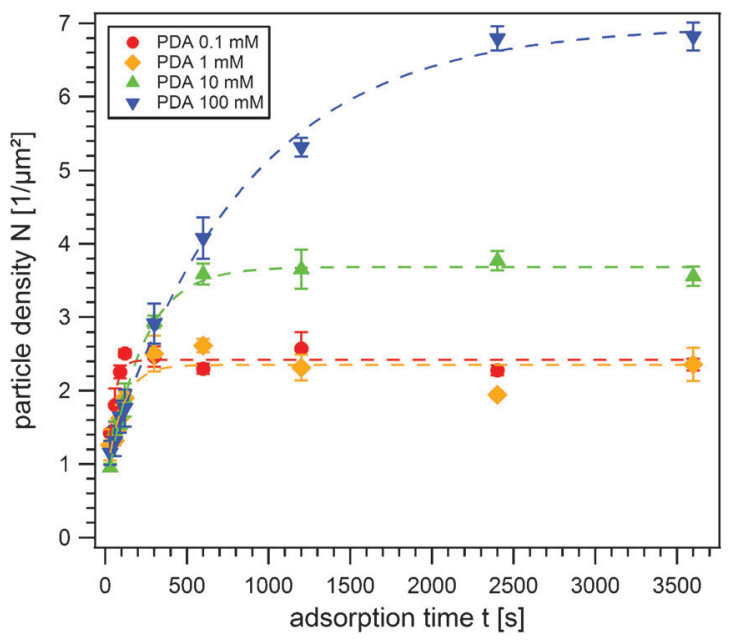

Fig. 3 Time dependence of SPB adsorption on PDA terminated polyelectrolyte multilayers. The saturation coverage strongly depends on the ionic strength and is reached in less than $60 \mathrm{~min}$. As a guide to the eye exponential functions were fitted to the data.

is observed at any concentration of added salt. Upon contact of the PSS brush with the PDA terminated multilayer counterions are released. ${ }^{20}$ Especially at low ionic strength the related gain in entropy causes a strong attractive interaction between the SPB and the surface. As shown for cationic SPB adsorbed on mica the force of adhesion strongly depends on the ionic strength. Complete detachment of SPB particles during AFM scanning was observed after raising the ionic strength to $10 \mathrm{mM} .{ }^{19}$ Interestingly, with increasing salt concentration we observed a shift of the saturation particle density towards higher coverage. At high salt concentrations the blocking effect of the negatively charged SPB adsorbed to the surface is significantly reduced. ${ }^{30}$

Regardless of the ionic strength, the SPB deposition process follows a well-defined time dependence. In the absence of
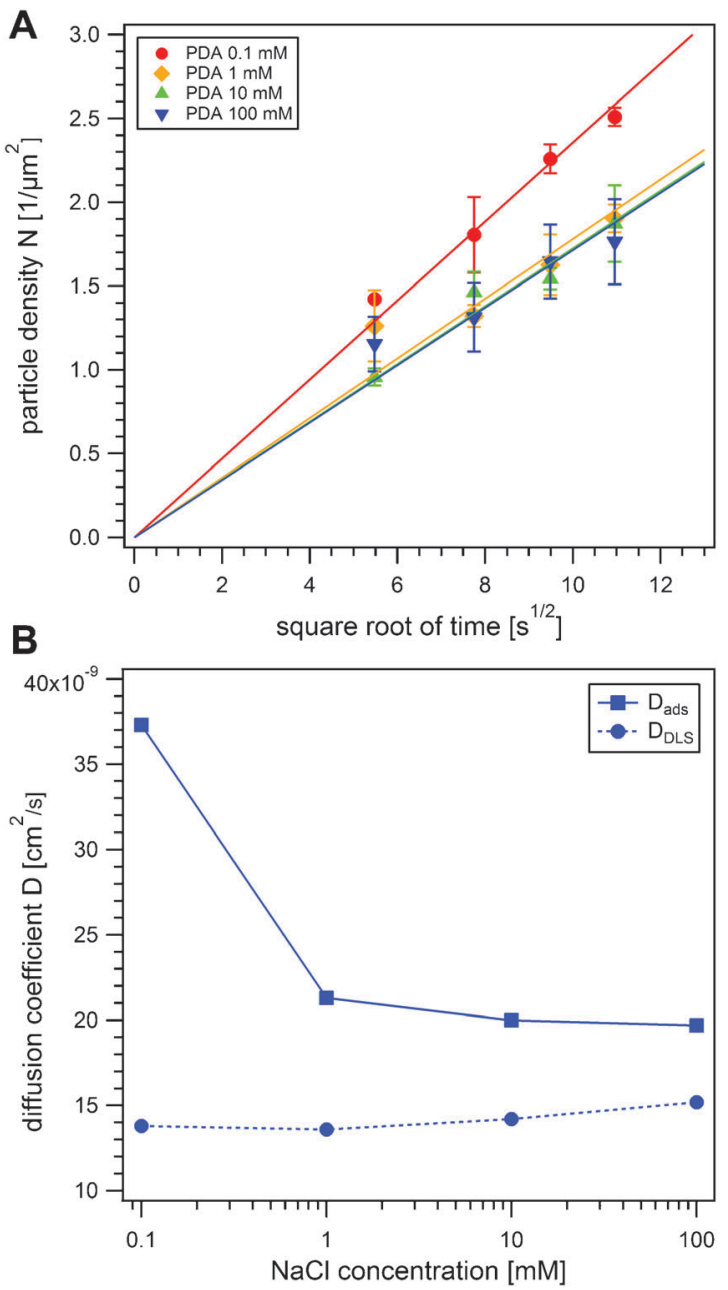

Fig. 4 The linear dependence of the particle density on the square root of time indicates a diffusion-limited process in the early adsorption stages (A). From the initial adsorption rates an apparent diffusion coefficient $D_{\text {ads }}$ can be calculated. With increasing ionic strength $D_{\text {ads }}$ approaches the diffusion coefficient $D_{\text {DLS }}$ measured by DLS (B).

hydrodynamic flows and blocking effects the flux of particles towards the substrate is expected to follow diffusion-limited kinetics. The evolution of the particle density $N(t)$ with time $t$ is then described by: ${ }^{31}$

$$
N(t)=2 c(D t / \pi)^{1 / 2}
$$

Here $c$ is the number concentration of SPB in the bulk phase, whereas $D$ represents the diffusion coefficient. Fig. 4A displays the first two minutes of the SPB adsorption process on PDA. The particle density $N$ is plotted as a function of the square root of time. In all cases linear regression through zero is possible confirming diffusion-limited initial adsorption kinetics. Remarkably, the adsorption rate at $0.1 \mathrm{mM}$ is $35 \%$ higher than those observed for higher salt concentrations. A similar rate dependence on the ionic strength has been reported for other highly charged particle systems, albeit for significantly lower concentrations of added salt. ${ }^{31}$ This effect can be explained by taking the presence of an oppositely charged wall into account. ${ }^{32}$

According to eqn (1) the initial adsorption rate $\mathrm{d} N(t) / \mathrm{d} t$ is linearly dependent on $c$ and $D^{1 / 2}$. During all the experiments 
Table 1 Diffusion coefficients calculated from the adsorption rates and measured by DLS for various ionic strengths

\begin{tabular}{llll}
\hline Substrate & $I / \mathrm{mM}$ & $D_{\mathrm{ads}} / \mathrm{cm}^{2} \mathrm{~s}^{-1}$ & $D_{\mathrm{DLS}} / \mathrm{cm}^{2} \mathrm{~s}^{-1}$ \\
\hline PDA & 0.1 & $3.73 \times 10^{-8}$ & $1.38 \times 10^{-8}$ \\
PDA & 1 & $2.13 \times 10^{-8}$ & $1.36 \times 10^{-8}$ \\
PDA & 10 & $2.00 \times 10^{-8}$ & $1.42 \times 10^{-8}$ \\
PDA & 100 & $1.97 \times 10^{-8}$ & $1.52 \times 10^{-8}$ \\
PSS & 100 & $1.66 \times 10^{-8}$ & $1.52 \times 10^{-8}$ \\
\hline
\end{tabular}

the particle concentration was kept constant at $0.1 \mathrm{wt} \%$. The variance of the adsorption rate must therefore be associated with a change of the apparent diffusion coefficient. The calculated diffusion coefficients $D_{\text {ads }}$ and the diffusion coefficients $D_{\text {DLS }}$ measured by dynamic light scattering are listed in Table 1 and plotted in Fig. 4B. All values are of the same order of magnitude, whereas the analysis of the adsorption rates yields slightly larger values for $D_{\text {ads }}$. It must be noted that the DLS measurements were carried out at substantially lower particle concentrations. However, viscometric data have shown that the DLS radii of SPB coincide with the hydrodynamic radii measured in the semidilute regime where the SPB start to interact. ${ }^{33}$
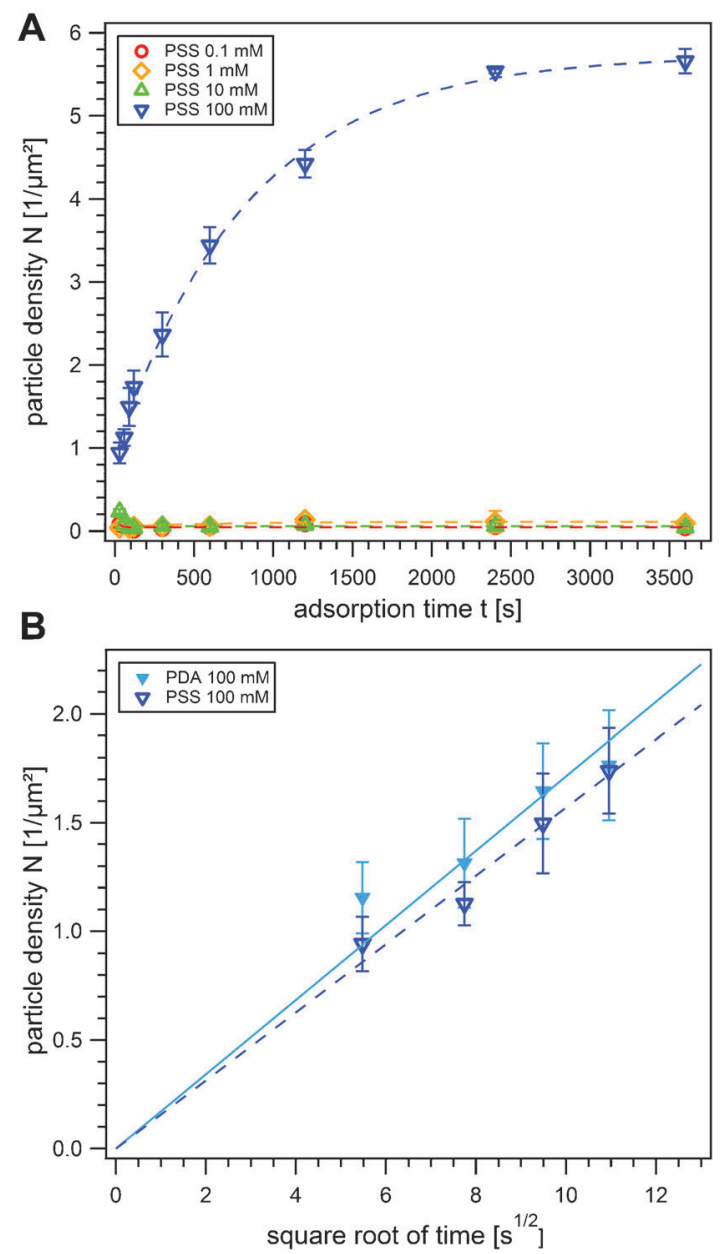

Fig. 5 The adsorption of SPB on PSS terminated multilayers is completely suppressed for salt concentrations up to $10 \mathrm{mM}$. At a high ionic strength of $100 \mathrm{mM}$ SPB deposition is facilitated by nonspecific secondary interactions (A). The initial adsorption kinetics on PSS is diffusion-limited and comparable to the one observed on PDA at $100 \mathrm{mM}$ (B).
Hence, the higher diffusivity found in the adsorption experiments must stem from the presence of an attractive wall. Screening of the electrostatic attraction among SPB and substrate evokes assimilation to the DLS data (Fig. 4B).

Further, the interaction of brush particles and equally charged substrates was probed. As displayed in Fig. 5, PSS terminated multilayers efficiently suppress SPB adsorption up to an ionic strength of at least $10 \mathrm{mM}$. Obviously, the amount of SPB remaining on the aqueous film after rinsing the substrate is negligible. However, at a salt concentration of $100 \mathrm{mM}$ the SPB adsorption kinetics follows the same trend on PSS as seen for PDA surfaces. Here the ionic strength of the bulk solution is roughly corresponding to the ionic strength inside the brush, i.e. the polyelectrolyte brush starts to resemble a neutral polymer brush. ${ }^{14}$ In this regime of strong electrostatic screening, the repulsion between the SPB in solution and the PSS multilayer, respectively, SPB already adsorbed on the PDA layer starts to vanish. Instead nonspecific interactions such as van der Waals or hydrophobic interaction become dominant and facilitate particle attachment. At an ionic strength of $100 \mathrm{mM}$, the adsorption kinetics on PSS is comparable to that observed on PDA. As displayed in Fig. 5B both substrates exhibit diffusion-limited initial kinetics with similar rates of adsorption. However, one cannot necessarily assume such kinetics for the adsorption onto PSS terminated PEM at concentrations of added salt lower than $100 \mathrm{mM}$. The substantially decreased sticking probability could govern the adsorption rate in this regime.

\section{Surface morphology and saturation coverage}

While the initial kinetics is diffusion-limited, for longer adsorption times surface blocking effects become dominant. In this regime, the adsorption of colloidal particles is often described by random sequential adsorption (RSA). ${ }^{34,35}$ The RSA model assumes stepwise particle deposition. Adsorption attempts are considered successful, if no geometric overlap between incoming and already adsorbed particles occurs. The fractional surface coverage $\theta$ increases with the number of adsorption steps until the jamming coverage $\theta_{\text {jam }}$ is reached. For non-interacting hard spheres with radius $R$ and an ideal collector surface Monte Carlo simulations predict a maximum surface coverage of $54.7 \%$. Considering charged particles a significantly lower maximum coverage $\theta_{\max }$ is common, as the interparticle repulsion leads to an increased effective radius commonly expressed by: ${ }^{30,31}$

$$
\begin{gathered}
\theta=N \pi R^{2} \\
\theta_{\max }=\theta_{\text {jam }}\left(R / R_{\mathrm{eff}}\right)^{2}
\end{gathered}
$$

where $R_{\text {eff }}$ is a function of the ionic strength.

The SPB system under investigation follows this general trend. At small salt concentrations the average interparticle distance clearly exceeds the hydrodynamic diameter. However, with rising ionic strength the amount of adsorbed particles increases steadily. Fig. 6A displays films prepared at $\mathrm{NaCl}$ concentrations ranging from $10 \mathrm{mM}$ to $500 \mathrm{mM}$ that were imaged before drying. The particles form non-close packed layers on the substrates, which typically result from random sequential adsorption. The liquid-like nature of these films is 

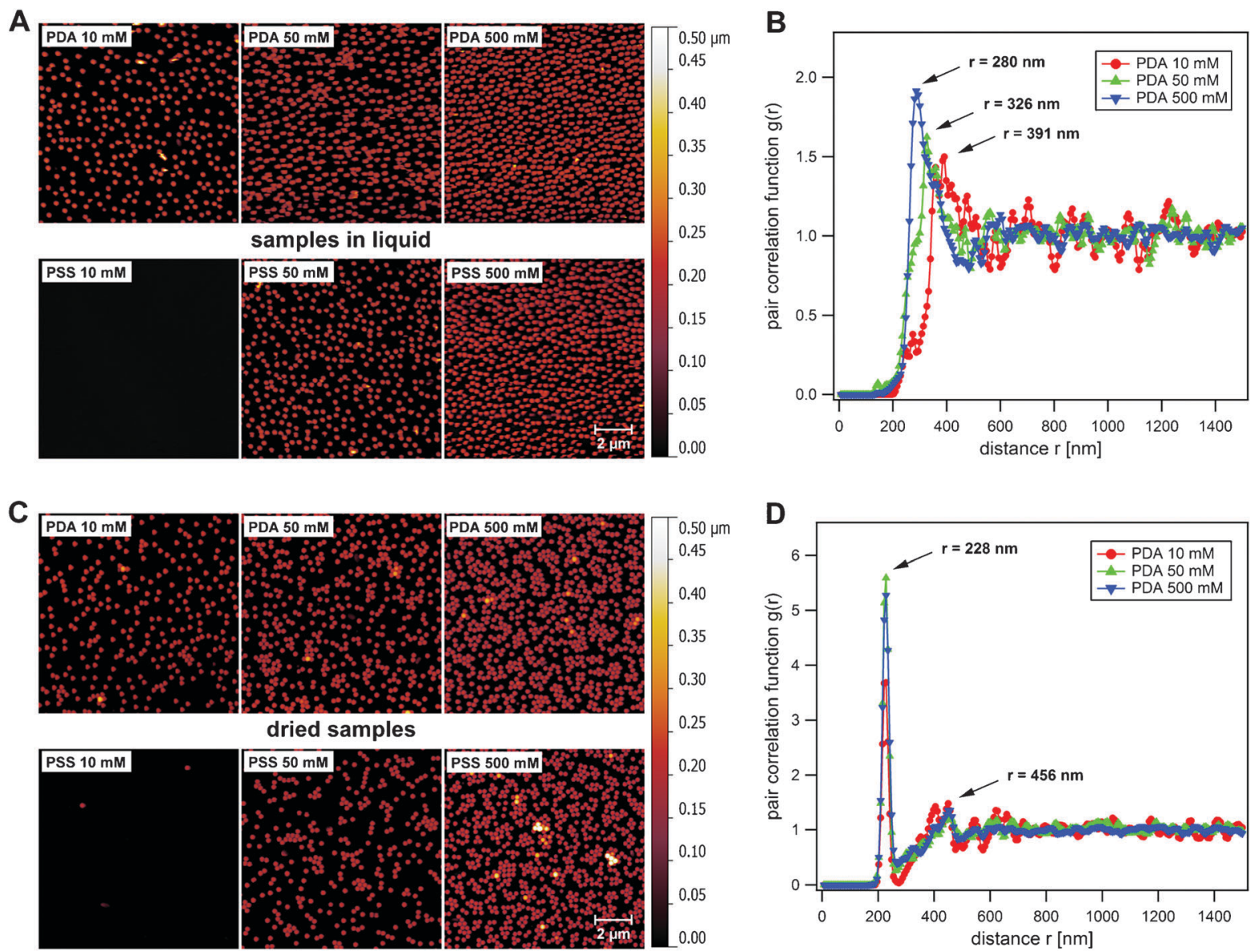

Fig. 6 Influence of substrate charge and ionic strength on thin film morphology. Before drying the films exhibit a random particle distribution with local density fluctuations (A). Increasing the ionic strength promotes adsorption on both types of substrates. The pair correlation functions of the corresponding PDA films show a broad peak, which is shifted to lower distances with increasing ionic strength (B). Capillary forces lead to the formation of densely packed islands during drying $(C)$. The corresponding pair correlation functions for the dried PDA samples display sharp maxima (D). The positions are independent of the salt concentration during deposition.

also reflected in pair correlation functions of the particle positions as depicted in Fig. 6B for PDA terminated multilayers. Due to local density fluctuations only a single broad peak is visible for each sample. The peak position is correlated with the distance between neighbouring particles and clearly shifts to lower values with increasing ionic strength.

Upon drying, the surface morphology changes from randomly distributed single particles to separated islands of several particles as shown in Fig. 6C. Attractive capillary forces lead to the formation of close-packed two-dimensional SPB arrays. This effect is most pronounced at high ionic strength where the surface coverage is high. Pair correlation functions of such dried samples display sharp peaks for the nearest and less pronounced peaks for the second nearest neighbours (Fig. 6D). The characteristic distance between the nearest neighbours is independent of the ionic strength highlighting the lateral mobility of SPB during drying. Most importantly, we found nearly identical particle coverage for wet and dry samples prepared under the same conditions. The values are listed in Table 2. For this reason, particle desorption during drying can be neglected independent of salt concentration and surface charge.

Fig. 7 displays the evolution of the maximum surface coverage $\theta_{\max }$ with increasing ionic strength. The maximum
Table 2 Comparison of the particle density before $\left(N_{\text {in situ }}\right)$ and after $\left(N_{\text {ex situ }}\right)$ drying

\begin{tabular}{llll}
\hline Substrate & $I / \mathrm{mM}$ & $N_{\text {in situ }} / \mu \mathrm{m}^{-2}$ & $N_{\text {ex situ }} / \mu \mathrm{m}^{-2}$ \\
\hline PDA & 10 & 3.58 & 3.56 \\
PDA & 50 & 5.47 & 5.61 \\
PDA & 500 & 8.58 & 9.00 \\
PSS & 10 & 0.01 & 0.05 \\
PSS & 50 & 4.05 & 3.78 \\
PSS & 500 & 8.52 & 9.15 \\
\hline
\end{tabular}

coverage $\theta_{\max }$ is calculated from the particle densities observed after an adsorption period of $60 \mathrm{~min}$. While the surface coverage on PDA increased monotonically with the ionic strength, virtually no adsorption occurred on PSS terminated substrates below a threshold salt concentration of $10 \mathrm{mM}$. Higher ionic strength leads to a gradual loss of substrate selectivity. However, matching surface coverage on both oppositely and like-charged substrates is found only at salt concentrations exceeding $100 \mathrm{mM}$. This particular value corresponds to the transition from the osmotic brush to the salted brush regime. ${ }^{11}$ Interestingly, previous studies showed that the binding of proteins to SPB is also strongly correlated with the transition from the osmotic to the salt brush regime. ${ }^{36}$ 


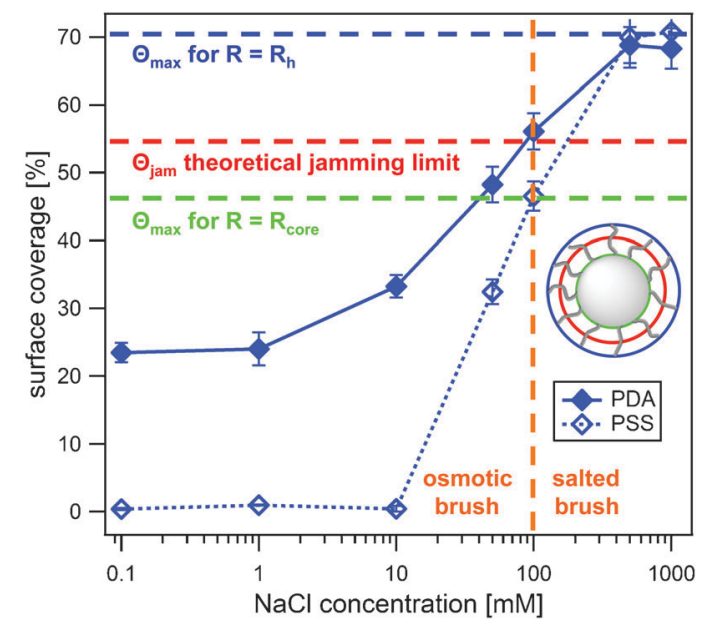

Fig. 7 The surface coverage on PDA increases monotonically with the ionic strength. For PSS terminated multilayers marked adsorption sets in between $10 \mathrm{mM}$ and $50 \mathrm{mM}$. At ionic strengths above $500 \mathrm{mM}$ the degrees of adsorption on PDA and PSS terminated PEM are consistent. The calculated maximum surface coverage depends on the assumed particle dimensions yielding $47 \%$ when only the core diameter is considered. Accounting for the shell thickness in solution a maximum coverage of $70 \%$ is found.

In the salted brush regime the polyelectrolyte corona is mostly collapsed and the SPB are expected to resemble the adsorption behaviour of uncharged hard spheres of the same hydrodynamic radius. Intriguingly, the observed maximum surface coverage exceeded the theoretical jamming limit of $54.7 \%$. At salt concentrations above $500 \mathrm{mM}$ the surface coverage reaches values around $70 \%$ independent of the terminating polyelectrolyte layer. Neglecting the polyelectrolyte corona completely a maximum coverage of $47 \%$ is found. Thus in experiments conducted at high ionic strength the observed effective radius lies between the measured hydrodynamic radius of the core and that of the core-shell particle. It is reasonable to assume deformations of the soft polyelectrolyte shell as the cause of this deviation from the RSA model.

\section{Conclusions}

We presented a systematic investigation of the adsorption behaviour of spherical polyelectrolyte brushes on polyelectrolyte multilayers. The adsorption kinetics was studied for positively and negatively charged top layers at ionic strengths ranging from $0.1 \mathrm{mM}$ to $100 \mathrm{mM}$. The adsorption was initially diffusion limited and gave way to an ionic strength dependent plateau value (saturation limit) with time. Hence, changing the concentration of added salt allowed us to precisely adjust the amount of deposited particles. The substrate can be switched from attracting to repelling the SPB by simply changing the sign of charge of the top layer at low concentrations of added salt. Given that oppositely charged polyelectrolytes interact, the gain in entropy by the concomitant release of counterions governs the interaction between the surface and the particles, whereas electrosteric repulsion results if substrate and particles carry the same sign of charge. With the transition from the osmotic to the salted brush regime at approximately $100 \mathrm{mM}$ attractive secondary interactions become dominant. At even higher ionic strength the maximum surface coverage exceeds the theoretical jamming limit. This deviation from the RSA model might be explained by a deformation of the soft polyelectrolyte shell in consequence of the adsorption.

Controlling the adsorption behaviour is a prerequisite for the production of multilayers and arrays of colloidal particles. In particular, the possibility for creating surfaces that are patterned in their charge density by polymer-on-polymer stamping ${ }^{37-39}$ provides the potential for controlling particle ordering on surfaces. While this has been demonstrated for hard particles ${ }^{39}$ and soft shells, ${ }^{40}$ the present system could allow for annealing structures because of the stimuli-responsive nature of the brush layer, which avoids an irreversible hit-stick behaviour. ${ }^{19}$ In addition, numerous chemical modifications have been reported for SPB particles ${ }^{15,18,41}$ and it has been demonstrated that the polyelectrolyte corona can be used as a well-defined reaction environment. ${ }^{42-44}$ Thus our study could open exiting avenues for the production of functional materials with hierarchical internal organization.

\section{Acknowledgements}

This work has been conducted within the special collaborative research project SFB 840 (project B5) and was funded by the German Science Foundation (DFG). The authors would like to thank Christoph Hasenöhrl and Mareen Müller for their help with the AFM imaging as well as Martin Hoffmann for guidance during the DLS measurements. We also thank Johann Erath for fruitful discussions.

\section{References}

1 G. Decher, Science, 1997, 277, 1232-1237.

2 G. Decher, J. D. Hong and J. Schmitt, Thin Solid Films, 1992, 210, 831-835.

3 S. T. Dubas and J. B. Schlenoff, Macromolecules, 1999, 32, 8153-8160.

4 D. Yoo, S. S. Shiratori and M. F. Rubner, Macromolecules, 1998, 31, 4309-4318.

5 A. Izquierdo, S. S. Ono, J. C. Voegel, P. Schaaf and G. Decher, Langmuir, 2005, 21, 7558-7567.

6 C. H. Porcel, A. Izquierdo, V. Ball, G. Decher, J. C. Voegel and P. Schaaf, Langmuir, 2005, 21, 800-802.

7 J. B. Schlenoff, S. T. Dubas and T. Farhat, Langmuir, 2000, 16, 9968-9969.

8 R. K. Iler, J. Colloid Interface Sci., 1966, 21, 569-594.

9 O. D. Velev and S. Gupta, Adv. Mater., 2009, 21, 1897-1905.

10 Y. N. Xia, B. Gates, Y. D. Yin and Y. Lu, Adv. Mater., 2000, 12, 693-713.

11 M. Ballauff, Prog. Polym. Sci., 2007, 32, 1135-1151.

12 X. Guo and M. Ballauff, Langmuir, 2000, 16, 8719-8726.

13 Y. Mei, K. Lauterbach, M. Hoffmann, O. V. Borisov, M. Ballauff and A. Jusufi, Phys. Rev. Lett., 2006, 97, 4.

14 X. Guo and M. Ballauff, Phys. Rev. E: Stat. Phys., Plasmas, Fluids, Relat. Interdiscip. Top., 2001, 64, 1-9.

15 A. N. Korovin, V. G. Sergeyev, O. A. Pyshkina, C. Hanske, A. Fery, A. Wittemann and L. Tsarkova, Macromol. Rapid Commun., 2011, 32, $462-467$

16 M. Schrinner, M. Ballauff, Y. Talmon, Y. Kauffmann, J. Thun, M. Möller and J. Breu, Science, 2009, 323, 617-620.

17 A. Wittemann and M. Ballauff, Phys. Chem. Chem. Phys., 2006, 8, 5269-5275.

18 Y. Lu, A. Wittemann and M. Ballauff, Macromol. Rapid Commun., 2009, 30, 806-815.

19 H. Gliemann, Y. Mei, M. Ballauff and T. Schimmel, Langmuir, 2006, 22, 7254-7259. 
20 Y. Mei, A. Wittemann, G. Sharma, M. Ballauff, T. Koch, H. Gliemann, J. Horbach and T. Schimmel, Macromolecules, 2003, 36, 3452-3456.

21 G. Decher and J. B. Schlenoff, Multilayer thin films: sequential assembly of nanocomposite materials, Wiley-VCH Verlag $\mathrm{GmbH} \&$ Co. KGaA, Weinheim, 2003.

22 W. S. Tan, R. E. Cohen, M. F. Rubner and S. A. Sukhishvili, Macromolecules, 2010, 43, 1950-1957.

23 W. S. Tan, Z. Zhu, S. A. Sukhishvili, M. F. Rubner and R. E. Cohen, Macromolecules, 2011, 44, 7767-7774.

24 J. Gensel, E. Betthausen, C. Hasenöhrl, K. Trenkenschuh, M. Hund, F. Boulmedais, P. Schaaf, A. H. E. Müller and A. Fery, Soft Matter, 2011, 7, 11144-11153.

25 L. Xu, Z. C. Zhu and S. A. Sukhishvili, Langmuir, 2011, 27, 409-415.

26 S. Kostler, A. V. Delgado and V. Ribitsch, J. Colloid Interface Sci., 2005, 286, 339-348.

27 M. Hoffmann, A. Jusufi, C. Schneider and M. Ballauff, J. Colloid Interface Sci., 2009, 338, 566-572.

28 W. Kern and D. A. Puotinen, RCA Rev., 1970, 31, 187-206.

29 A. Wittemann, M. Drechsler, Y. Talmon and M. Ballauff, J. Am. Chem. Soc., 2005, 127, 9688-9689.

30 R. Pericet-Camara, G. Papastavrou and M. Borkovec, Langmuir, 2004, 20, 3264-3270.

31 C. A. Johnson and A. M. Lenhoff, J. Colloid Interface Sci., 1996, 179, 587-599.
32 B. P. Cahill, G. Papastavrou, G. J. M. Koper and M. Borkovec, Langmuir, 2008, 24, 465-473.

33 A. Marra, E. Peuvrel-Disdier, A. Wittemann, X. Guo and M. Ballauff, Colloid Polym. Sci., 2003, 281, 491-496.

34 Z. Adamczyk, Adv. Colloid Interface Sci., 2003, 100, 267-347.

35 B. Senger, J. C. Voegel and P. Schaaf, Colloids Surf., A, 2000, 165, $255-285$.

36 A. Wittemann, B. Haupt and M. Ballauff, Phys. Chem. Chem. Phys., 2003, 5, 1671-1677.

37 X. P. Jiang, H. P. Zheng, S. Gourdin and P. T. Hammond, Langmuir, 2002, 18, 2607-2615.

38 M. Pretzl, A. Schweikart, C. Hanske, A. Chiche, U. Zettl, A. Horn, A. Böker and A. Fery, Langmuir, 2008, 24, 12748-12753.

39 H. P. Zheng, M. F. Rubner and P. T. Hammond, Langmuir, 2002, 18, 4505-4510.

40 M. Nolte and A. Fery, Langmuir, 2004, 20, 2995-2998.

41 Y. Lu, Y. Mei, R. Walker, M. Ballauff and M. Drechsler, Polymer, 2006, 47, 4985-4995.

42 B. Haupt, T. Neumann, A. Wittemann and M. Ballauff, Biomacromolecules, 2005, 6, 948-955.

43 Y. Lu, Y. Mei, M. Schrinner, M. Ballauff, M. W. Möller and J. Breu, J. Phys. Chem. C, 2007, 111, 7676-7681.

44 Y. Mei, Y. Lu, F. Polzer, M. Ballauff and M. Drechsler, Chem. Mater., 2007, 19, 1062-1069. 